\title{
The association of prolonged breastfeeding and allergic disease in poor urban children
}

\author{
C.C. Obihara*\#, B.J. Marais*, R.P. Gie*, P. Potter ${ }^{\ddagger}$, E.D. Bateman+, C.J. Lombard ${ }^{\S}$, \\ N. Beyers* and J.L.L. Kimpen ${ }^{\#}$
}

ABSTRACT: The fact that breastfeeding may protect against allergic disease remains controversial, with hardly any reports from developing countries. This study investigated the association between allergic disease in children and prolonged breastfeeding.

Data were collected from a $15 \%$ random sample of households from two poor suburbs of Cape Town, South Africa. Parents completed a validated International Study on Asthma and Allergies in Childhood questionnaire on allergic diseases for children aged 6-14 yrs. Other questions included breastfeeding duration, maternal smoking and parental allergy. Results were adjusted for possible confounders and for possible clustering within the household.

Out of the 861 children included in the study, allergic disease in general, and hay fever in particular, were significantly less frequent in those with prolonged ( $\geqslant 6$ months) breastfeeding. There was a significant linear inverse association between breastfeeding duration and allergic disease in children without allergic parents, but not in children with an allergic predisposition.

In conclusion, these results from a developing country suggest a protective effect of prolonged breastfeeding on the development of allergic disease, particularly hay fever, in children born to nonallergic parents. This protective effect was not found in children with an allergic predisposition.

KEYWORDS: Allergic disease, children, prolonged breastfeeding

$\mathbf{T}$ he prevalence of allergic disease in developed countries has shown a dramatic increase in recent decades, and the relationship between breastfeeding and allergic disease in these countries remains uncertain. In developing countries, allergic disease continues to rise [1], and the relationship between breastfeeding and allergic disease has not been evaluated.

The increasing prevalence of allergic disease has been linked to reduced microbial exposure in early life $[2,3]$. Specific microbial stimuli are thought to stimulate T-helper (Th)-1 immune responses, thus reducing the activity of the atopyassociated Th-2 response [3]. Breast milk influences the bacterial flora in the infant gut [4], which may provide an important immune stimulus in infancy [5]. The possible protective effect of breastfeeding on allergic disease remains controversial and epidemiological studies have yielded variable results [6-9], with little information from developing countries where the infection pressure is higher.

The current authors set out to test the hypothesis that prolonged breastfeeding duration (based on the World Health Organization (WHO) definition), in children from a poor urban setting in a developing country, would be associated with less allergic disease than a shorter breastfeeding duration.

\section{MATERIALS AND METHODS \\ Study setting}

The current study was conducted in two suburbs (total population 38,565 in 1996) of Cape Town, South Africa, with poor socio-economic conditions (average household income US\$ 98.77). The population density was high (6-14 occupants per household), but all households had running water and electricity. A previous International Study on Asthma and Allergies in Childhood (ISAAC) survey carried out in Cape Town, within a similar age group, had estimated the prevalence of different allergic disease manifestations as the following: asthma $13.3 \%$, allergic rhinoconjunctivitis $16 \%$ and eczema $9 \%$ [10]. In accordance with WHO recommendations, mothers are routinely encouraged to practice prolonged breastfeeding. This is based on the generally accepted benefits of breast milk against serious childhood infections, such as gastroenteritis.

\section{AFFILIATIONS}

${ }^{*}$ Centre for TB Research and Education (CENTRE), Dept of Paediatrics and Child Health, Faculty of Health Sciences, Stellenbosch University, Stellenbosch,

-Allergy Diagnostic and Clinical

Research Unit, and

+Division of Pulmonology, Dept of Medicine, University of Cape Town, and

${ }^{\S}$ Biostatistics Unit, Medical Research Council, Cape Town, South Africa \#Wilhelmina Children's Hospital, University Medical Center, Utrecht, The Netherlands.

CORRESPONDENCE

C.C. Obihara

Centre for TB Research and

Education (CENTRE)

Dept of Paediatrics and Child Health

Faculty of Health Sciences

Stellenbosch University

P0 Box 19063

South Africa

Fax: 27219389138

E-mail: obiha000@sun.ac.za

Received:

October 082004

Accepted after revision:

April 052005

SUPPORT STATEMENT

C.C. Obihara is the recipient of a grant from the Ter Meulen Fund, Royal Netherlands Academy of Arts and Sciences, Amsterdam, The Netherlands. This study was partly funded through a research grant from the South African Dept of Trade and Industry (THRIP fund), Pretoria, South Africa.

European Respiratory Journal

Print ISSN 0903-1936

Online ISSN 1399-3003 


\section{Sample selection}

A 15\% sample of household addresses was randomly selected. Trained fieldworkers enumerated the people at each selected household, and all children aged 6-14 yrs were invited to participate in the study. Written informed consent was obtained from a parent or legal guardian before study enrolment.

\section{Study design}

A cross-sectional, questionnaire-based survey was conducted from July-December, 2002, in children aged 6-14 yrs.

\section{Questionnaire}

Parents completed a questionnaire for each child under the supervision of a trained fieldworker. Questions on allergic disease were based on the validated ISAAC phase-I questionnaire, translated into the local language (Afrikaans). Questions were asked on both previous (ever) and recent ( $\leqslant 12$ months) asthma, hay fever and eczema. Additional questions included: breastfeeding duration; maternal smoking during pregnancy and current household exposure to cigarette smoke; number of siblings; maternal education; household income; allergic history of the mother and father; and previous serious infectious illnesses (upper and lower respiratory tract infections, gastroenteritis, urinary tract infections, ear, nose and throat infections, measles, flu and other viral infections) other than tuberculosis (TB), for which the child had to visit a doctor, a health centre or be admitted to a hospital. Data on breastfeeding duration were collected as a categorical variable as follows: breastfed $<6$ months, $6-12$ months or $>12$ months.

\section{Tuberculin skin test}

The burden of TB is very high in the study area. The average notification rate of new bacteriologically confirmed TB is 320 per $100,000 \cdot \mathrm{yr}^{-1}$ [11]. A positive tuberculin skin test (TST) is a hallmark of primary infection with Mycobacterium tuberculosis (MTB) [12]. TST reactivity becomes apparent 3-6 weeks after initial MTB infection and may remain positive for the lifetime of the individual [12]. As MTB is known to stimulate a Th-1 immune reaction [3], the TST response was documented in the children. Experienced study nurses carried out the TST by injecting $2 \mathrm{TU}$ of purified protein derivative RT 23 (Statens Serum Institut, Copenhagen, Denmark) intradermally on the volar aspect of the left forearm. The largest transverse diameter of induration was recorded after 2-3 days. In accordance with the American Thoracic Society guidelines, a positive TST was defined as an induration of $\geqslant 10 \mathrm{~mm}$ [12].

\section{Definitions}

Allergic disease in general refers to the presence of at least one allergic disease: hay fever, asthma or eczema. Prolonged breastfeeding is defined as breastfeeding for $>6$ months, and included all children that were breastfed, irrespective of the exclusivity of breastfeeding.

\section{Statistical analysis}

The Chi-squared test was used to calculate associations between different allergic diseases and the duration of breastfeeding, and the Chi-squared test for linear-by-linear association was used to detect linear trends. Logistic regression (LR) analyses were performed with a random-effects LR model, using different allergic disease phenotypes as the dependent variable and breastfeeding duration as the independent variable. Adjustment was made for potential confounding variables such as demographic (age and sex), socio-economic (average household income and number of siblings), genetic (parental allergic history) and environmental factors (maternal smoking during pregnancy and TST response). Results were corrected for possible clustering in the sampling unit. The sampling unit in this study (cluster survey) was the household. In total, there were 500 households in the current study, with an average of 1.6 children per household. For the analysis, each individual child was coded and linked to the specific household. By using the randomeffects LR model for the multivariate analyses, correction was made for the possibility that more than one child came from the same household.

The study received ethics approval from the University of Stellenbosch (Stellenbosch, South Africa) and the relevant local authorities.

\section{RESULTS}

Out of the 978 children enumerated, 884 (90.4\%) consented to participation in the study. Of these, 861 children $(97.4 \%)$ with data on breastfeeding duration were included in the analysis. The sex distribution was equal and the mean \pm SD age was $10.4 \pm 2.5$ yrs. Since only $2.6 \%$ (23) of the consenting children were never breastfed, the small size of this group provided insufficient power for a separate analysis; together with the fact that the study question focused on association of breastfeeding duration and allergic disease, these children were excluded from the analysis. The baseline demographic parameters did not differ in those children who were excluded.

Table 1 describes the most important characteristics of the 861 children analysed. Out of the total number of children, 381 $(44.3 \%)$ were breastfed for $>6$ months. Children exposed to a short or prolonged breastfeeding duration did not differ with respect to age, sex, maternal education level, maternal or paternal allergic history, maternal smoking during the pregnancy or positive TST (table 1). Neither did they differ in the reported frequency of previous serious infections other than TB $(p=0.25)$, nor rate of hospital admissions $(p=0.40)$. However, children who received prolonged breastfeeding were more likely to come from low-income households and to have $\geqslant 3$ siblings (table 1 ).

Figure 1 illustrates the differences in frequency of different allergic disease phenotypes between children exposed to a short or prolonged breastfeeding duration. The risk of allergic disease in general, and hay fever in particular, was significantly lower in children with prolonged breastfeeding than in those with short breastfeeding duration (adjusted odds ratio (OR): $0.50 ; 95 \%$ confidence interval (CI): $0.31-0.82$ and adjusted OR: 0.53 ; 95\% CI: $0.29-0.99$, respectively), while this was not statistically significant for asthma (adjusted OR: 0.67; 95\% CI: 0.31-1.49) or eczema (OR: 0.56; 95\% CI: 0.29-1.08).

There was a significant linear inverse association between the breastfeeding duration and the frequency of allergic disease in general, and hay fever in particular, but not for asthma or eczema (table 2). 
TABLE 1 Baseline characteristics of children with short ( $<6$ months) and prolonged ( $\geqslant 6$ months) breastfeeding duration

\begin{tabular}{|c|c|c|c|c|c|}
\hline & Total & $\begin{array}{l}\text { Breastfeeding } \\
<6 \text { months }\end{array}$ & $\begin{array}{c}\text { Breastfeeding } \\
\geqslant 6 \text { months }\end{array}$ & $\begin{array}{l}\text { Unadjusted OR } \\
\qquad(95 \% \mathrm{Cl})\end{array}$ & $\begin{array}{c}\text { Adjusted OR } \text { OR }^{\#} \\
(95 \% \mathrm{Cl})\end{array}$ \\
\hline \multicolumn{6}{|l|}{ Age yrs } \\
\hline $6-8$ & 281 & $160(56.9)$ & $121(43.1)$ & 1.0 & 1.0 \\
\hline 9-14 & 580 & $320(55.2)$ & $260(44.8)$ & $1.07(0.80-1.40)$ & $1.03(0.75-1.40)$ \\
\hline Female & 423 & $231(54.6)$ & $192(45.4)$ & $1.09(0.83-1.43)$ & $1.05(0.79-1.40)$ \\
\hline \multicolumn{6}{|c|}{ Socio-economic parameters } \\
\hline \multicolumn{6}{|c|}{ Maternal education yrs spent at school } \\
\hline$<7$ & 443 & $235(53.0)$ & $208(47.0)$ & 1.0 & 1.0 \\
\hline$\geqslant 7$ & 404 & $235(58.2)$ & $169(41.8)$ & $0.81(0.62-1.06)$ & $0.93(0.69-1.24)$ \\
\hline \multicolumn{6}{|l|}{ Household income } \\
\hline$<3$ & 600 & $363(60.5)$ & $237(39.5)$ & 1.0 & 1.0 \\
\hline$\geqslant 3$ & 234 & $100(42.7)$ & $134(57.3)$ & $2.05(1.51-2.79)$ & $1.82(1.31-2.54)$ \\
\hline \multicolumn{6}{|c|}{ Positive parental allergic history } \\
\hline \multicolumn{6}{|c|}{ Mother } \\
\hline No & 699 & $396(56.7)$ & $303(43.3)$ & 1.0 & 1.0 \\
\hline Yes & 162 & $84(51.9)$ & $78(48.1)$ & $1.22(0.86-1.71)$ & $1.37(0.94-1.99)$ \\
\hline \multicolumn{6}{|l|}{ Father } \\
\hline No & 752 & $419(55.7)$ & $333(44.3)$ & 1.0 & 1.0 \\
\hline Yes & 109 & $61(56.0)$ & $48(44.0)$ & $0.99(0.66-1.48)$ & $0.99(0.63-1.54)$ \\
\hline \multicolumn{6}{|c|}{ Maternal smoking during the pregnancy } \\
\hline No & 463 & $255(55.1)$ & $208(44.9)$ & 1.0 & 1.0 \\
\hline Yes & 394 & $222(56.3)$ & $172(43.7)$ & $0.95(0.73-1.25)$ & $0.87(0.64-1.17)$ \\
\hline
\end{tabular}

Data are presented as $\mathrm{n}(\%)$, unless otherwise stated. OR: odds ratio; Cl: confidence interval; TST: tuberculin skin test. \#: breastfeeding duration was used as a dependent variable and the other variables were used as independent variables in the random-effects logistic regression model, which corrected for possible bias due to clustering in the sample unit; ": an average household income of $<800$ South African Rand (SAR) was used to differentiate low-income households (800 SAR is the official minimum monthly wage; 1 US $\$=6.5$ SAR).

The relationship between different categories of breastfeeding duration and allergic disease phenotypes was evaluated in a random-effects LR model, together with possible confounding variables. In this model, the risk of different allergic diseases was compared between group 1 (breastfed $<6$ months), group 2 (6-12 months) and group 3 (>12 months). Compared with group 1, group 2 had significantly less risk of allergic disease in general (adjusted OR: 0.52; 95\% CI: 0.28 0.97 ), while group 3 had significantly less risk of allergic disease in general, and hay fever in particular. While the difference in risk of allergic disease in general between group 2 and group 1 was no longer observed after the introduction of the interaction factor between breastfeeding duration and maternal allergic history, the difference in risk of allergic disease in general and hay fever between group 3 and group 1 remained significant (table 3).

Due to the strong effect of maternal allergic history in the LR model, a stratified analysis was performed based on this variable. Table 4 shows the crude stratified analysis of the linear trend between different categories of breastfeeding duration and allergic diseases in children with and without a maternal or paternal allergic history. In children without a maternal or paternal allergic history, there was a significant linear inverse association between the breastfeeding duration and allergic disease in general, and hay fever in particular, with the maternal history having the stronger influence. However, in children with a maternal or paternal allergic history, no such linear inverse association was found. There was a significant interaction factor between maternal allergic history and breastfeeding duration for allergic disease in general $(p=0.02)$ and asthma in particular $(p=0.03)$, but not for hay fever $(p=0.3)$ or eczema $(p=0.5)$. Stratified LR analysis based on maternal allergic history showed that maternal allergic history seems to modify the effect of breastfeeding duration on allergic disease (table 5). In children without a maternal allergic history, the risk of allergic disease in general 


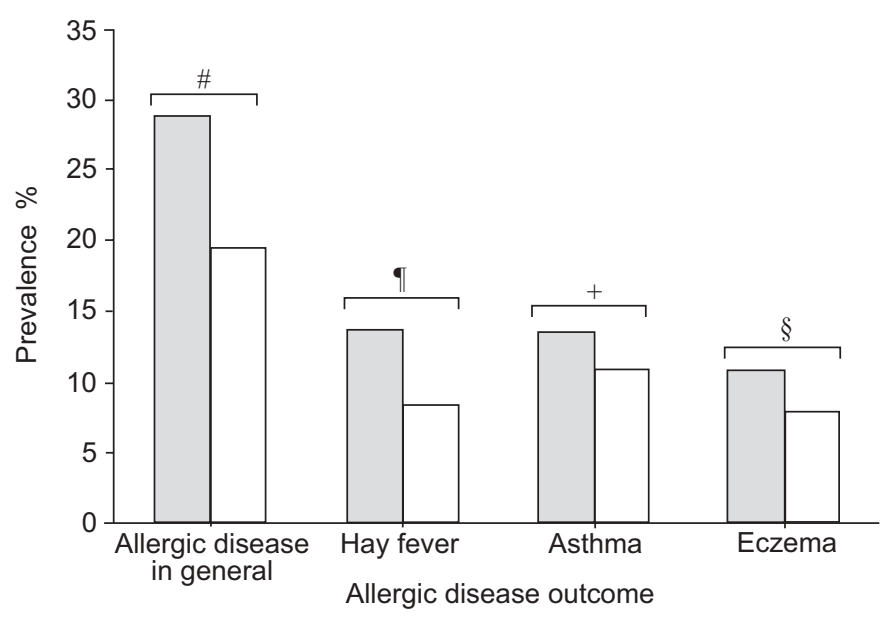

FIGURE 1. Prevalence of allergic disease phenotypes in children with short ( $<6$ months; $\square$ ) and prolonged ( $\geqslant 6$ months; $\square$ ) breastfeeding duration. Allergic disease in general: $n=213$; hay fever: $n=98$; asthma: $n=106$; eczema: $n=80$. \#: $p=0.005 ;$ : $p=0.04{ }^{+}: p=0.2 ;{ }^{\varsigma}: p=0.08$.

\section{TABLE 2}

Frequency distribution of reported allergic disease phenotypes for different categories of breastfeeding duration showing the linear trend analysis

\begin{tabular}{|c|c|c|c|c|c|}
\hline & \multicolumn{5}{|c|}{ Breastfeeding duration } \\
\hline & $\begin{array}{c}\text { Total } \\
\mathbf{n}\end{array}$ & $\begin{array}{c}<6 \\
\text { months }\end{array}$ & $\begin{array}{c}\text { 6-12 } \\
\text { months }\end{array}$ & $\begin{array}{c}\quad>12 \\
\text { months }\end{array}$ & p-value $\#$ \\
\hline Subjects $n$ & & 480 & 153 & 228 & \\
\hline Hay fever & 98 & $66(13.8)$ & $16(10.5)$ & $16(7.0)$ & 0.008 \\
\hline Asthma & 106 & 65 (13.5) & $15(9.9)$ & $26(11.5)$ & 0.35 \\
\hline Eczema & 80 & $51(10.7)$ & $10(6.6)$ & $19(8.4)$ & 0.25 \\
\hline Allergic disease & 213 & 139 (28.9) & $31(20.3)$ & $43(18.9)$ & 0.002 \\
\hline
\end{tabular}

Data are presented as $\mathrm{n}$ or $\mathrm{n}(\%)$, unless otherwise stated. ${ }^{\#}$ : Chi-squared test for linear trend was used to compare differences in prevalence of different allergic diseases for different categories of breastfeeding duration $(p \leqslant 0.05$ signifies a statistically significant trend in association); ${ }^{\natural}$ : in general

decreased progressively with longer duration of breastfeeding, being significant for those breastfed $>12$ months. In contrast, in children with maternal allergic history, no such decrease in risk was observed (table 5). A similar trend was observed for asthma, although not significant.

\section{DISCUSSION}

The results of this study found a significant inverse association between prolonged breastfeeding (based on WHO definition) and the prevalence of allergic disease, which suggests a protective effect of breastfeeding on the development of allergy in these children living in a poor socio-economic area within a developing country. This protective effect was most pronounced for hay fever, which may reflect the fact that hay fever is the allergic disease that is most strongly associated with atopy [13].

The mechanisms responsible for the protective effect of breastfeeding against allergic disease are not well understood, but several factors may contribute. The hygiene hypothesis suggests that reduced microbial exposure in childhood results in reduced Th- 1 activity, with resultant Th- 2 dominance and an increased incidence of allergy [2, 3, 14]. Breast milk stimulates, through its high oligosaccharide contents, intestinal colonisation with specific bacterial flora, such as lactobacilli, bifidobacteria and nonpathogenic Escherichia coli, which may influence the development of gut-driven immune responses [4, 5, 15-18]. Gut colonisation with these bacteria induces the production of Th-1 cytokines, which counterbalance Th-2 activity [5]. This is supported by studies which have shown that allergic children are less frequently colonised with lactobacilli and bifidobacteria than nonallergic children [19, 20]. In addition, breast milk may provide direct protection against allergic sensitisation through decreased exposure to food antigens, by enhanced maturation of the intestinal mucosal barrier and via immunoglobulins (Ig), such as secretory $\operatorname{Ig} \mathrm{A}$, secreted in the milk $[18,21]$.

A striking feature of the results is the observation of an inverse association of breastfeeding duration and the risk of allergic disease in children without an allergic predisposition. A history of allergy in either parent appeared to neutralise the protective effect of prolonged breastfeeding. This observation has been reported previously [22], but the study evaluated a select group of hospitalised patients whose mothers were encouraged to carry on breastfeeding as long as possible. Differences in the breast milk content between allergic and nonallergic mothers have been proposed as a possible explanation for this observation, because the breast milk of allergic mothers has lower levels of ovalbumin-specific IgA, salivary $\operatorname{IgA}$, alpha-linoleic and polyunsaturated fatty acids, and higher levels of Th-2 promoting cytokines [23-26]. However, the fact that the inverse association between prolonged breastfeeding and allergic disease was negated by an allergic family history, irrespective of whether it was maternal or paternal, suggests that genetic predisposition is probably the overriding factor. Even though a history of maternal allergy was associated with an increased risk of allergic disease in the children in the current study, this did not influence the association of prolonged breastfeeding and allergic disease in the multivariate analysis.

A few important characteristics differentiate the present study from the less recent ones [6-9, 22]. First, this study was conducted in a socio-economically disadvantaged urban community, within a developing country. Secondly, more than a quarter of the children were breastfed for $>12$ months, which enabled the current authors to analyse the results of prolonged breastfeeding in two categories (6-12 months and $>12$ months). Thirdly, a random, community-based sampling method, without pre-selection of particular groups where the healthcare provider or researcher could have biased the choice or duration of infant feeding method, was used.

An interesting observation was the association found between maternal smoking during pregnancy and the risk of asthma in 


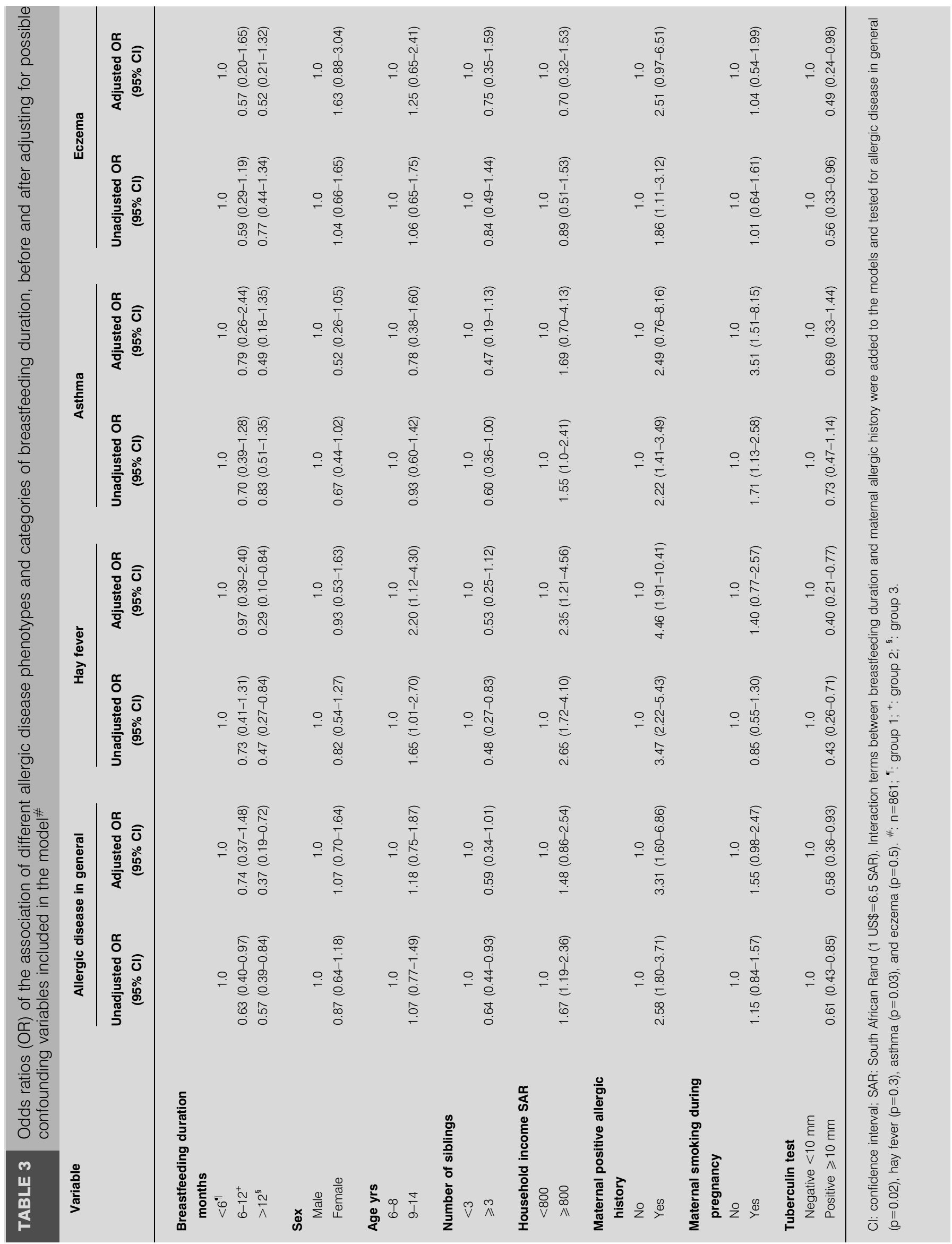


TABLE 4 Crude stratified linear association between breastfeeding duration and different allergic diseases in children based on parental allergic history

\begin{tabular}{|c|c|c|c|c|c|}
\hline Parental history & $\begin{array}{c}\text { Breastfeeding } \\
\text { duration months }\end{array}$ & $\begin{array}{l}\text { Allergic disease } \\
\text { in general }\end{array}$ & Hay fever & Asthma & Eczema \\
\hline \multirow{2}{*}{ Allergic mother } & $6-12$ & $22.6(7 / 31)$ & $16.1(5 / 31)$ & $10.0(3 / 30)$ & $6.5(2 / 31)$ \\
\hline & $>12$ & $44.7(21 / 47)$ & $21.3(10 / 47)$ & $30.4(14 / 46)$ & $17.4(8 / 46)$ \\
\hline$p$-value & & 0.7 & 0.3 & 0.2 & 0.9 \\
\hline Allergic father & $>12$ & $44.4(12 / 27)$ & $22.2(6 / 27)$ & $38.5(10 / 26)$ & $19.2(5 / 26)$ \\
\hline p-value & & 0.4 & 0.2 & 0.3 & 1.0 \\
\hline \multirow{3}{*}{ Nonallergic mother } & $<6$ & $25.5(101 / 396)$ & $10.6(42 / 396)$ & $12.4(49 / 395)$ & $9.7(38 / 392)$ \\
\hline & $6-12$ & $19.7(24 / 122)$ & $9.0(11 / 122)$ & $9.9(12 / 121)$ & $6.6(8 / 121)$ \\
\hline & $>12$ & $12.2(22 / 181)$ & $3.0(6 / 181)$ & $6.7(12 / 180)$ & $6.1(11 / 179)$ \\
\hline $\mathrm{p}$-value & & $<0.001$ & 0.005 & 0.04 & 0.1 \\
\hline$p$-value & & 0.003 & 0.02 & 0.1 & 0.2 \\
\hline
\end{tabular}

Data are presented as \% $(n / N)$, unless otherwise stated. Chi-squared test for linear trend was used to compare the prevalence of different allergic diseases. Allergic disease in general implies at least one allergic disease (hay fever, asthma or eczema). $p \leqslant 0.05$ signifies a statistically significant linear inverse association.

TABLE 5 Odds ratios (OR) showing effect modification of the association of allergic disease and breastfeeding duration by maternal allergic history\#

\begin{tabular}{lccccc} 
& \multicolumn{2}{c}{ Allergic disease in general } & & Asthma \\
\cline { 2 - 3 } $\begin{array}{l}\text { Breastfeeding } \\
\text { duration months }\end{array}$ & $\begin{array}{c}\text { No maternal } \\
\text { allergic history }\end{array}$ & $\begin{array}{c}\text { Maternal } \\
\text { allergic history }\end{array}$ & & $\begin{array}{c}\text { No maternal } \\
\text { allergic history }\end{array}$ & $\begin{array}{c}\text { Maternal } \\
\text { allergic history }\end{array}$ \\
\hline $\mathbf{6}$ & 1.0 & 1.0 & & 1.0 & 1.0 \\
$\mathbf{6}-\mathbf{1 2}$ & $0.71(0.35-1.44)$ & $0.28(0.08-0.91)$ & & $0.55(0.11-2.76)$ & $0.27(0.03-2.06)$ \\
$>\mathbf{1 2}$ & $0.36(0.18-0.73)$ & $1.27(0.53-3.02)$ & & $0.43(0.11-1.65)$ & $3.57(1.00-12.79)$ \\
\hline
\end{tabular}

Data are presented as OR (95\% confidence interval). The analysis was limited to the two allergic disease phenotypes with a significant interaction coefficient with maternal allergic history in the logistic regression (LR) analysis (table 3). The random-effects LR model was used, with allergic disease phenotype as a dependent variable, and breastfeeding duration and confounding variables as independent variables. Results were corrected for possible bias due to the clustering in the sampling unit. \#: adjusted for possible confounding variables: demographic (age and sex), socio-economic (number of siblings and household income), genetic predisposition (maternal allergic history was used in the model because its effect was stronger than paternal allergic history), and environmental exposures (maternal smoking during the pregnancy and tuberculin skin test reactivity)

the child; this was not influenced by present smoking exposure. This is consistent with results from developed countries [27-29], which demonstrate that maternal smoking remains an important environmental risk factor for the development of asthma in children, even in poor settings.

The present study does have several limitations inherent to its cross-sectional design and the retrospective questionnairebased diagnosis. These include recall bias, reporter subjectivity and the fact that allergic disease was self-reported. Using the validated ISAAC-based questionnaire reduced some of the limitations associated with questionnaire-based evaluation.
The possibility that health workers may have exerted a biased influence on the mother's decision to prolong breastfeeding depending on the perceived risk of allergy in the child seems unlikely, as the frequency of maternal allergic history did not differ between children with prolonged breastfeeding duration and those with short breastfeeding duration. Even though the presence of a positive TST was independently associated with a reduced risk of allergic disease, it did not influence the association found between breastfeeding duration and allergic disease in the multivariate analysis. The reported prevalence of childhood infections other than MTB was also similar in the different breastfeeding groups. There is a possibility that the 
lack of association of breastfeeding duration and previous infection between the groups analysed may be related to the fact that all previous infections were analysed as a single variable. However, this does not seem to influence the results, as the frequency did not differ between the different categories of breastfeeding duration.

The current authors were unable to analyse the results of children who were not breastfed; the statistical power was limited by the small size of this group. Data on exclusive breastfeeding were not available, which made it impossible to differentiate between the effects of exclusive breastfeeding and mixed feeding, but it is known that few mothers in the study area breastfeed exclusively [30].

The World Health Organization promotes prolonged breastfeeding (for $\geqslant 6$ months) as the preferred method of infant feeding, especially in settings with poor healthcare services and unhygienic living conditions, to reduce serious infections in infancy [31]. An additional advantage of promoting prolonged breastfeeding as a public health strategy may be the reduction of allergic disease, especially hay fever, in children without an allergic predisposition. There is a need for prospective community-based studies to evaluate the relation of breastfeeding duration and atopy.

\section{ACKNOWLEDGEMENTS}

The authors would like to thank all parents and children who participated; I. Toms (Director of Health, City of Cape Town, South Africa) for permission to conduct the study in the community; and S. Schaaf, C. Lawrence and S. Den Boon from the Dept of Paediatrics, Stellenbosch University (South Africa), for helping with data analysis and manuscript correction.

\section{REFERENCES}

1 Weinberg EG. Urbanization and childhood asthma: an African perspective. J Allergy Clin Immunol 2000; 105: 224-231.

2 Strachan D. Hay fever, hygiene and household size. BMJ 1989; 299: 1259-1260.

3 Shirakawa T, Enomoto T, Shimazu S, Hopkin J. The inverse association between tuberculin responses and atopic disorder. Science 1997; 275: 77-79.

4 Yashioka H, Iseki K, Fujita F. Development and differences of intestinal flora in neonatal period of breast-fed and bottle-fed infants. Pediatrics 1983; 72: 317-321.

5 Hessle C, Hanson A, Wold AE. Lactobacilli from human gastrointestinal mucosa are strong stimulators of IL-12 production. Clin Exp Immunol 1999; 116: 276-282.

6 Saarinen UM, Kajosaari M, Backman A, Siimes MA. Prolonged breastfeeding as prophylaxis for atopic disease. Lancet 1979; 2: 163-166.

7 Saarinen UM, Kajosaari M. Breastfeeding as prophylaxis against atopic diseases: prospective follow-up study until 17 years old. Lancet 1995; 346: 1065-1069.

8 Wright AL, Holberg CJ, Taussig LM, Martinez FD. Relationship of infant feeding to recurrent wheezing at age 6 years. Arch Pediatr Adolesc Med 1995; 149: 758-763.

9 Sears MR, Greene JM, Willan AR, et al. Long-term relation between breastfeeding and development of atopy and asthma in children and young adults: a longitudinal study. Lancet 2002; 360: 901-907.

10 The International Study of Asthma and Allergies in Childhood (ISAAC) Steering Committee. Worldwide variation in prevalence of symptoms of asthma, allergic rhinoconjunctivitis, and atopic eczema: ISAAC. Lancet 1998; 351: 1225-1232.

11 Verver S, Warren RM, Munch Z, et al. Proportion of tuberculosis transmission that takes place in households in a high-incidence area. Lancet 2004; 363: 212-214.

12 Shingadia D, Novelli V. Diagnosis and treatment of tuberculosis in children. Lancet Infect Dis 2003; 3: 624-632.

13 Pearce N, Pekkanen J, Beasley R. How much asthma is really attributable to atopy? Thorax 1999; 54: 268-272.

14 Rook G, Stanford J. Give us this day our daily germs. Immunol Today 1998; 19: 113-116.

15 Sepp E, Julge K, Vasar M, Naaber P, Björkstén B, Mikelsaar M. Intestinal microflora of Estonian and Swedish infants. Acta Paediatr 1997; 86: 956-961.

16 Lodinová R, Jouja V. Antibody production by mammary gland in mothers after artificial oral colonization of their infants with non-pathogenic strain E. coli. Acta Paediatr Scand 1977; 66: 705-708.

17 Prescott S, Macaubas C, Holt B, et al. Transplacental priming of the human immune system to environmental allergens: universal skewing of initial $\mathrm{T}$ cell responses toward the Th2 cytokine profile. J Immunol 1998; 160: 4730-4737.

18 Hoppu U, Kalliomäki M, Laiho K, Isolauri E. Breast milk immunomodulatory signals against allergic diseases. Allergy 2001; 56: Suppl. 67, 23-26.

19 Björkstén B, Naaber P, Sepp E, Mikelsaar M. The intestinal microflora in allergic Estonian and Swedish 2-year-old children. Clin Exp Allergy 1999; 29: 342-346.

20 Kalliomäki M, Salminen S, Arvilommi H, Kero $\mathrm{P}$, Koskinen P, Isolauri E. Probiotics in primary prevention of atopic disease: a randomised placebo-controlled trial. Lancet 2001; 357: 1076-1079.

21 Brandtzaeg P. Current understanding of gastrointestinal immunoregulation and its relation to food allergy. Ann NY Acad Sci 2002; 964: 13-45.

22 Savilahti E, Tainio V-M, Salmenperä L, Siimes MA, Perheentupa J. Prolonged exclusive breastfeeding and heredity as determinants in infantile atopy. Arch Dis Child 1987; 62: 269-273.

23 Casas R, Böttcher MF, Duchén K, Björkstén B. Detection of $\operatorname{IgA}$ antibodies to cat, $\beta$-lactoglobulin, and ovalbumin allergens in human milk. J Allergy Clin Immunol 2000; 105: 1236-1240.

24 Van Asperen PP, Gleeson M, Kemp AS. The relationship between atopy and salivary IgA deficiency in infancy. Clin Exp Immunol 1985; 62: 753-757.

25 Duchén K, Casas R, Böttcher MF, Yu G, Björkstén B. Human milk polyunsaturated long-chain fatty acids and secretory immunoglobulin A antibodies and early childhood allergy. Pediatr Allergy Immunol 2000; 11: 29-39.

26 Böttcher MF, Jenmalm MC, Garofalo RP, Björkstén B. Cytokines in breast milk from allergic and nonallergic mothers. Pediatr Res 2000; 47: 157-162. 
27 Martinez FD, Cline M, Burrows B. Increased incidence of asthma in children of smoking mothers. Pediatrics 1992; 89: 21-26.

28 Cook DG, Strachan DP. Health effects of passive smoking10: summary of effects of parental smoking on the respiratory health of children and implications for research. Thorax 1999; 54: 357-366.
29 Cook DG, Strachan DP, Carey IM. Parental smoking and spirometric indices in children. Thorax 1998; 53: 884-893.

30 Van der Elst CW, Pick W, Isaacs S, Malan AF. Current trends in infant feeding. S Afr Med J 1989; 76: 434-437.

31 Black RE, Victoria CG. Optimal duration of exclusive breastfeeding in low income countries. BMJ 2002; 325: $1252-1253$. 